\title{
What will come after the $\mathrm{Z}^{0}$ ?
}

\section{CERN now seems confident that it has observed five examples of the $Z^{0}$ particle, a kind of heavy photon. What is there left to do?}

IT'S official! CERN, the European centre for nuclear research, has discovered the $\mathrm{Z}^{0}$ - the particle related to the photon, but heavier, which mediates the "neutral current" component of the weak nuclear force. The $\mathrm{Z}^{0}$, with its charged partners the $\mathrm{W}^{+}$and $\mathrm{W}^{-}$, discovered together at CERN earlier this year, was predicted by the Salem-Weinberg model. This model unifies the weak and electromagnetic forces in a single field theory.

A few weeks ago, evidence was found of the production and decay of just one $Z^{0}$, but the evidence was somewhat equivocal and no official announcement was made (see Nature 19 May p.193). Now there are five examples, one of the "a text-book version", according to Professor Peter Kalmus of Queen Mary College, London, one of the 130-man, 13-university research team that discovered the particles.

This "UA1" collaboration works with the proton-antiproton collider at CERN, a device which uses the Super Proton Synchronotron (SPS) to accelerate and store protons moving in one direction and antiprotons in the other. Where the beams collide, many kinds of particles are created, among them Ws and Zs.

UA1 now has four $Z^{0}$ s decaying into an electron and a positron, and one into muon (heavy electron) and antimuon. The mass appears to be "about" $95 \mathrm{GeV}$ - less than the figure rumoured for the first event, and more in line with the Salem-Wienberg model, in relation to the $81 \pm 2 \mathrm{GeV}$ quoted in a recent UA1 seminar for the mass of the $\mathrm{W}^{-}$. The group has roughly 30 examples of production and decay of the $\mathrm{W}$.

Even so, it seems, there will be no published masses until the present datataking run is complete on 4 July, when the detector will be calibrated. By then the group would like to have $60-70 \mathrm{~W}$ decays and 6-7 $Z^{0}$ decays in the bag, with conclusions on masses and fits to the Salem-Weinberg theory by July.

The theory predicts $\mathrm{W}$ and $\mathrm{Z}$ masses which depend on a number called the "Weinberg angle", which measures quantum mechanical mixing between the raw photon and $\mathrm{Z}^{0}$ and their physical counterparts. Expressed as $\sin ^{2} \theta_{w}$, the presently accepted value is $0.23 \pm 0.01$. Using this number, the Salem-Weinberg theory yields a $W$ mass of $82 \pm 2$. On the same basis, the theory predicts a $\mathrm{Z}$ mass of around $93 \mathrm{GeV}$, consistent so far with UA1 estimates.

UAI measurements also show that the W decays are asymmetric, as should be the case because of the weak interaction's violation of the "parity" between right and left. In the $\mathrm{W}^{+}$decays found at CERN, for example, the positron in the decay tends to move off in the same hemisphere as the antiproton, as predicted by the theory.

Professor Abdus Salam, speaking from the International Centre for Theoretical Physics in Trieste this week, described himself as "very delighted" with the results. "I have the greatest admiration for the people involved", he said, "and particularly for the construction of the collider".

Salam sees these results in one respect as "the end of the road". Most of the grand unified theories that go beyond the SalamWeinberg model include colour - the force field of the quarks, which are the components of particles like protons and are forced to invoke phenomena at extremely high energies (or equivalently tiny distances) to explain the splitting between the fundamental forces we observe. These energies (of $10^{19} \mathrm{GeV}$ or so) are in practice

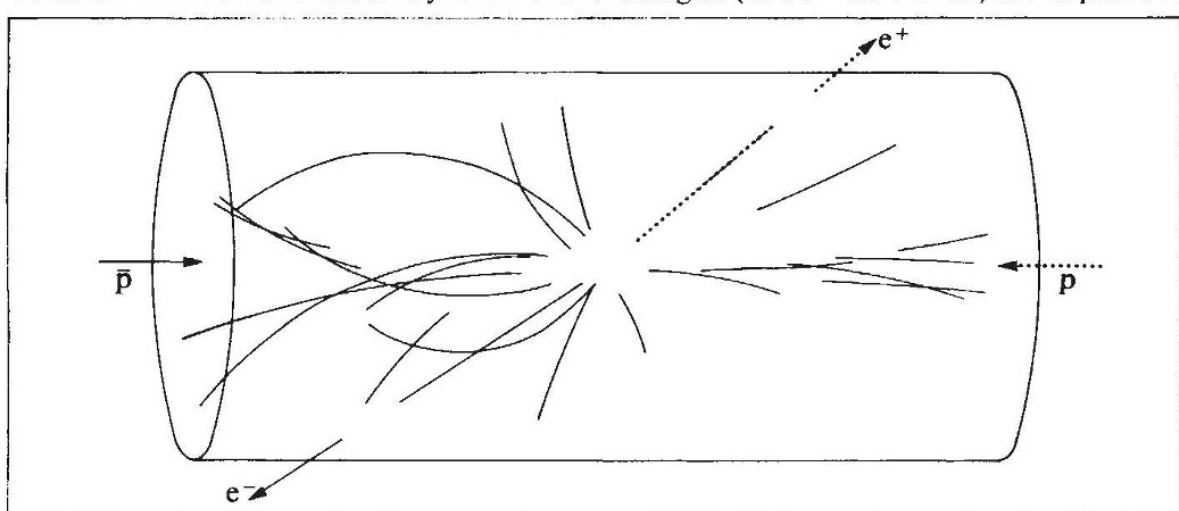

Collision of a proton and antiproton at the centre of CERN's 6-metre image chamber. Straight tracks are those of an electron and positron (dotted line) from the decay of the short-lived $Z^{\prime \prime}$.

unobtainable with present technologies, so that theories must be tested indirectly, by measurement of the decay of the proton and the like.

However, Salam has another theory, with his collaborator Pati, in which the leftright asymmetry of the weak force is easier to understand ... This could be tested with the CERN collider, Salam says. It predicts a second $\mathrm{Z}^{0}$, at higher mass (above $200 \mathrm{GeV}$ ), which the CERN collider should produce about one time for every fifty times it produces an ordinary $Z^{0}$. The UAl group could detect it if they could collect enough events - which means increasing the "luminosity" of the colliding beams.

Such an increase is indeed on the cards. According to Kalmus "we had a few events [not including Ws and Zs] in 1981; we had 10 times the number in 1982; we might get another factor of 10 this year, and if we get more running time, even another factor of $5-10$ the year after". This might be enough to see Salam's second $Z^{0}$. If not, there are plans to improve the production and collection of antiprotons, which is one of the main limitations on the collider luminosity, by building a new collector ring. But this would take three years to build, and take money from CERN's next big project, the large electron-positron collider LEP. This latter is due to produce physics in mid-1989, but since it is to be constructed on a constant (and in real terms slightly reducing) CERN budget, money spent on the collector might delay LEP. Salam's opinion: "I think Rubbia [the collider designer and leader of UA1] should be given every possible help".

According to Kalmus, there is also plenty to do with the collider other than search for Salam's putative second $\mathrm{Z}^{0}$ : study jets, for example, where a quark emerges from a proton-antiproton collision virtually isolated but then - as quarks cannot exist alone - "dresses itself" in a cloud of other strongly interacting particles, emerging thus as a clump of tracks isolated from others in the events. These jets may hide evidence of heavy quarks (a sixth, called the top quark, is needed to complete the present list of five). They may also conceal "Higgs particles", whose mass is unknown but whose existence is ultimately crucial to the Salam-Weinberg model. Another possibility is to search for Centauro events, seen a few times in cosmic rays, which indicate that a new kind of physics may set in at energies not much above present values.
Robert Walgate 